\title{
Effect of Propagation Signal and Path on Verification Performance Using Intra-Body Propagation Signals
}

\author{
Isao Nakanishi \\ Tottori University \\ 4-101 Koyama-Minami \\ Tottori, Tottori 680-8552, Japan \\ nakanishi@eecs.tottori-u.ac.jp \\ Ryutaro Nishi \\ Tottori University \\ 4-101 Koyama-Minami \\ Tottori, Tottori 680-8552, Japan
}

\author{
Itaru Ogushi \\ Tottori University \\ 4-101 Koyama-Minami \\ Tottori, Tottori 680-8552, Japan
}

\author{
Takahiro Murakami \\ Tottori University \\ 4-101 Koyama-Minami \\ Tottori, Tottori 680-8552, Japan
}

\begin{abstract}
Biometrics is the verification or the identification method of users by measuring and analyzing their biometric data, which is only applicable to continuous authentication in a system. In particular, unconsciously presentable biometric modalities are also applicable to an authentication system. As such a biometrics, to use intra-body propagation signals that propagate on a body surface as electromagnetic waves have been proposed. In conventional approaches, verification performance on palms has been evaluated by a white signal as a propagation signal. In this paper, it is reported that the effects of using a synthesized signal by sinusoidal waves with fixed amplitudes and phases instead of the white signal and propagating this signal on other body parts on verification.
\end{abstract}

\section{CCS CONCEPTS}

- Security and privacy $\rightarrow$ Biometrics;

\section{KEYWORDS}

Biometrics, Intra-body propagation signal, Propagation signal, Propagation path

\section{ACM Reference format:}

Isao Nakanishi, Itaru Ogushi, Ryutaro Nishi, and Takahiro Murakami. 2017. Effect of Propagation Signal and Path on Verification Performance Using Intra-Body Propagation Signals. In Proceedings of ICBEA '17, Hong Kong, Hong Kong, April 21-23, 2017, 5 pages.

DOI: http://dx.doi.org/10.1145/3077829.3077838

\section{INTRODUCTION}

Using biometrics system in our daily life has become very popular over the past two decades [4]. User authentication by the fingerprint for cellular phones is very common nowadays. This usage assumes

Permission to make digital or hard copies of all or part of this work for personal or classroom use is granted without fee provided that copies are not made or distributed for profit or commercial advantage and that copies bear this notice and the full citation on the first page. Copyrights for components of this work owned by others than ACM must be honored. Abstracting with credit is permitted. To copy otherwise, or republish, to post on servers or to redistribute to lists, requires prior specific permission and/or a fee. Request permissions from permissions@acm.org.

ICBEA '17, Hong Kong, Hong Kong

(C) 2017 ACM. 978-1-4503-4871-3/17/04 .. \$15.00

DOI: http://dx.doi.org/10.1145/3077829.3077838 one-time-only authentication. However, the one-time-only authentication has a fatal flaw on the point of view of user management. Once a genuine user is authenticated and remove all the barriers, anyone can use the system instead of the genuine user.

In order to prevent such an identity theft, continuous or successive (on-demand) authentication is needed $[1,5]$. In particular, unconsciously (transparently) presented biometric data to the system is needed to realize continuous or successive authentication. If the biometric data is presented consciously, users must keep presenting biometric data during continuous authentication or users must present biometric data whenever authentication is required in successive authentication. These degrade the usability of the system. The password and the ID card are unable to perform continuous or successive authentication because of the similar reason. Continuous or successive authentication is realized only by using unconsciously presentable biometrics.

Unconsciously presentable biometrics can be classified into two types. One of the types is passively measured biometrics data such as face and ear. However, biometric data of the face or the ear can be captured easily by using sensors (cameras). In other words, it is easy for others to steal biometric data while users are unaware of being captured. As a result, the fake faces or ears can be made by using captured data and then used for spoofing. The other is biometrics that is detectable from continuous action of users, for example, the voiceprint in speaking, gait in walking, keystroke in typing and so on. However, they can be usable only in their actions; therefore, their applicable situations are limited. In conclusion, conventional biometric modalities are unsuitable for continuous or successive authentication.

Intra-body propagation signal has been proposed to use as biometrics [8-10]. Verification performance was evaluated using propagated signals on forearms. In recent years, considering applications of authentication using intra-body propagation signals, verification performance using propagated signals on palms was evaluated $[3,6,7]$. However, their verification performances were not satisfied. One reason is that a white (random) signal was used as a source signal. A white signal is a signal that statistically contains even spectral elements. It is useful for us to detect spectral distribution in propagated signals. All spectral elements can be measured in a short time. However, the spectral uniformness of the source signal is not guaranteed in such a short time. In this paper, a synthesized 


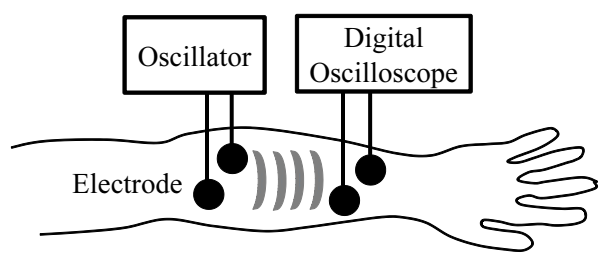

Figure 1: Intra-body propagation signal.

signal by sinusoidal waves with fixed amplitudes and phases is used as a source signal. This definite signal may improve the stability of a spectral characteristic of propagated signals and it may result in improvement on verification performance.

Another reason comes from places of a body where source signals are propagated. While verification performances on forearms and palms were evaluated in conventional studies, other body places may be suitable for person verification using intra-body propagation signals. In this paper, verification performances using propagated signals between one's right-hand palm and one's right-hand wrist, left-hand palm, right ear, or left-foot ankle are evaluated.

\section{INTRA-BODY PROPAGATION SIGNAL}

When a source signal is passed through an electrode pair on a body surface, an electrical field is generated around the electrode pair on the body surface and then it is detected as a voltage change at another electrode pair. These are described in Fig. 1. We treat the voltage change as an intra-body propagation signal. If the characteristics of propagation signals are different from user to user, then the propagation signals can be used as biometrics. This is the motivation of our study.

In the conventional studies [3, 6-10], a source signal was a quasiwhite Gaussian signal with a range of $100 \mathrm{MHz}$ from a signal oscillator. A signal detector is a digital oscilloscope with $200 \mathrm{MHz}$ frequency range, $1 \mathrm{GSa}$ /s sampling rate and memory size of 32 Mpts. Propagated signals digitized in the digital oscilloscope are transferred to a computer and analyzed by using FFT. Power spectra of propagated signals were used as individual features in person verification.

The reason why a white signal is used as a source signal is to obtain propagation characteristics efficiently. It is well known that a white signal contains even spectral elements. This issue makes it easy to extract all spectral elements. When a source signal has such characteristic, detecting spectral distribution in a propagated signal corresponds to detecting a frequency characteristic of a propagated path on a body. However, such spectral uniformness in a white signal is statistically guaranteed; therefore, it is not guaranteed in a short measuring time. Through many years of our research on intra-body propagation signals, it becomes aware that spectral nonuniformity in a short measuring time may cause degradation in verification performance. In this paper, a definite signal as a source signal instead of a random (white) signal. The definite signal is obtained by synthesizing sinusoidal waves with fixed amplitudes and phases. Further explanations and results are described in Sec. 4.

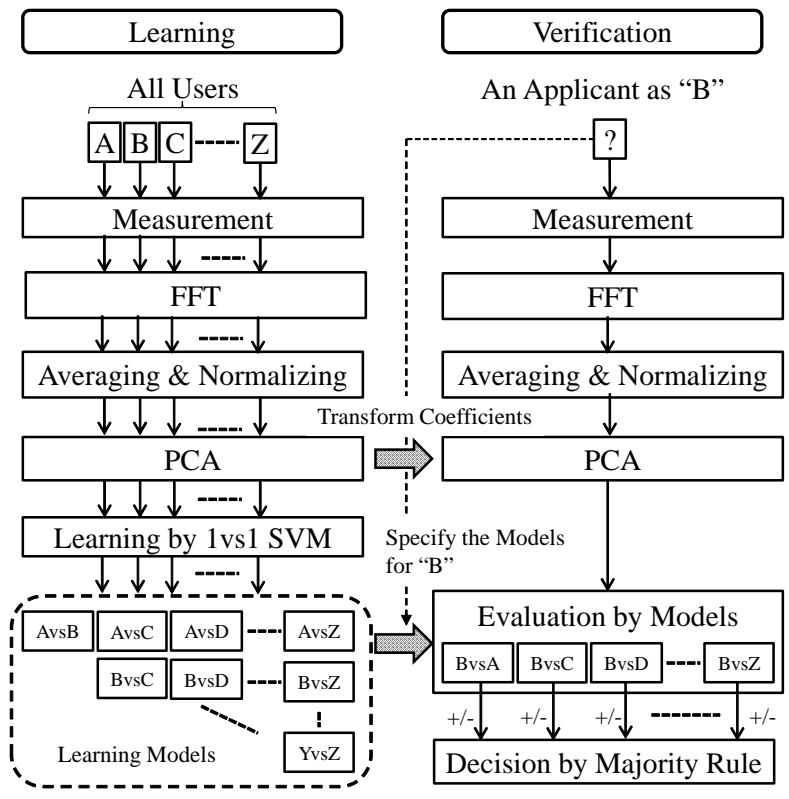

Figure 2: A procedure of verification using intra-body propagation signals.

Another problem is a body part where signals are propagated. In Refs. [8, 10], signals which propagated on forearms were measured using general-purpose electrodes and evaluated their verification performance in order to confirm the feasibility of person verification using intra-body propagation signals In Refs. [3, 6, 7], propagated signals on palms were measured because human beings always control a system by gripping or touching any point of the system and palms become only an interface between human beings and the system. However, verification performances in the conventional studies are not satisfactory. Therefore, the following expectation arises: signals that propagate on other body parts may be suitable for person verification. Further explanations and results are described in Sec. 5.

\section{VERIFICATION METHOD}

The procedure of verification using intra-body propagation signals is described in Fig. 2. In the learning stage, intra-body propagation signals are measured from all of the users in a system and then transformed to their spectra by using FFT. After spectral smoothing and normalizing, principal component analysis (PCA) is applied to the spectra. PCA works as a transformation from spectra to principal components while reducing the number of information dimensions. By setting the value of accumulated proportion in PCA, the number of principal components is determined. Transformation coefficients for obtaining principal components are memorized in the system. By using accumulated loadings, frequency elements that are related to principal components are extracted and they are regarded as effective frequency elements for user verification and thus used as individual features. By learning such individual features, in 


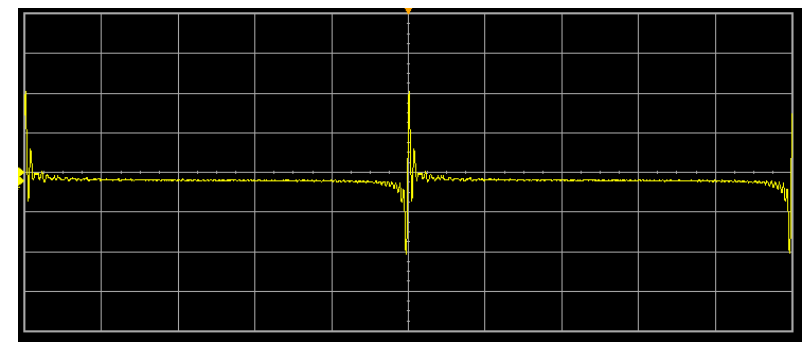

Figure 3: Waveform of a source signal.

support vector machine (SVM), models for distinguishing a target user from another user are obtained. This learning method is called one versus one SVM (1vs1SVM) (1vs1SVM) [2]. 1vs1SVM models are learned by teaching ' +1 ' for data from target users and ' -1 ' for data from others.

In the verification stage, an applicant who seeks the permission for using the system presents the name or the ID number of a user. From the applicant, an intra-body propagation signal is measured and smoothed, and a normalized spectrum is obtained through the same process as in the learning stage. From the spectrum, spectral elements that are at effective frequencies for verification are extracted and evaluated in learned SVM models that are related to the user whom the applicant specified. Each 1vs1SVM model outputs a positive value or a negative value. If the number of models with the positive output value is bigger than a threshold, that is, based on a majority rule, the applicant is recognized as genuine

\section{EFFECT OF DEFINITE SOURCE SIGNAL}

In the conventional studies, a source signal was a white (random) signal with a range of $100 \mathrm{MHz}$. On the other hand, a definite signal is used, which is produced by synthesizing sinusoidal waves with $1,2, \cdots, 100 \mathrm{MHz}$ and zero phases. A waveform in two cycles is shown in Fig. 3. Its amplitude is $4 V_{P-P}$.

It is natural that the spectral uniformness and the stability of the proposed definite source signal are superior to those of a white signal in a short measuring time. In the following, we confirm this issue through evaluation of verification performance using propagated signals on palms.

\subsection{Measuring Devices}

For measuring propagated signals on palms that are called intrapalm propagation signals, three dedicated devices with different electrode positions were prepared and these are conveniently called 'S', 'M', and ' $L$ ' in the followings. The reason behind that is for adapting to different sizes of the palm. One of the devices is shown in Fig. 4. The base of the devices is made from silicon and the shape of the palm is modeled concavely. There are poles for guiding to put a palm in proper position on the devices. In Fig. 4, ' $\mathrm{A}$ ' is a distance between an electrode on the detector side and a guiding pole, ' $\mathrm{B}$ ' is a distance between electrodes on the source and the detector sides, and ' $\mathrm{C}$ ' is a distance between electrodes on the source side. Those values in three devices were decided based on statistical data of Japanese palms and are summarized in Table 1.

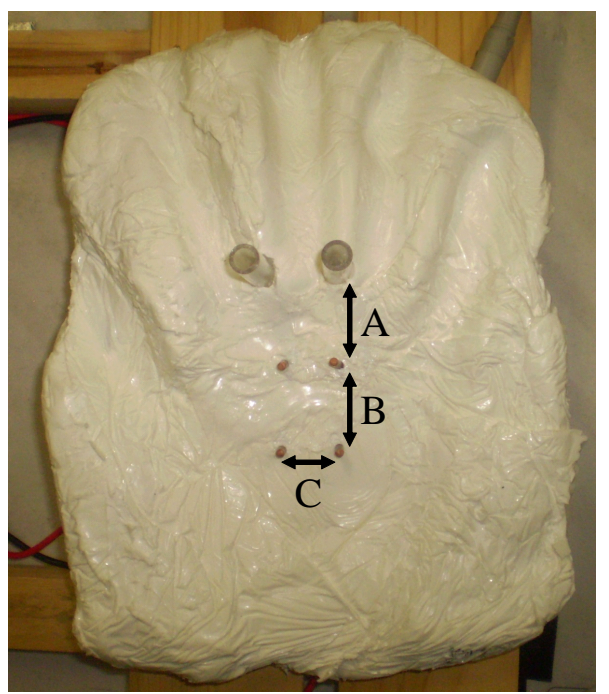

Figure 4: Distances: 'A', 'B', and 'C' in a measuring device.

Table 1: Dedicated measuring devices.

\begin{tabular}{c|c|c|c}
\hline Device Name & A $(\mathrm{mm})$ & B $(\mathrm{mm})$ & $\mathrm{C}(\mathrm{mm})$ \\
\hline $\mathrm{S}$ & 27 & 28 & 20 \\
\hline $\mathrm{M}$ & 29 & 30 & 20 \\
\hline $\mathrm{L}$ & 31 & 32 & 20 \\
\hline
\end{tabular}

\subsection{Measurement Conditions}

The number of experimental subjects was fifteen. They all were male students from our university. Palm size of each experimental subject was measured and suitable one was allocated to him/her from three devices considering his/her palm size.

Propagation signals were measured using a digital oscilloscope from each subject twice a day keeping an interval of at least four hours and repeated ten times (days). The sampling rate of the oscilloscope was $200 \mathrm{MHz}$.

Before each measuring, all subjects were required to wipe their palms by using moist tissues. Then, subjects sit a chair and put their palms on a measuring device that had been already allocated according to their palm sizes. During measuring, subjects were required to be relaxed and kept from moving.

\subsection{Performance Evaluation}

Verification performance was examined when using a definite source signal in order to verify its effectiveness. In PCA, the number of principal components was one by setting accumulated proportion to $41.2 \%$. By using accumulated loadings from the one principal component, frequency elements that were related to the principal component were extracted. As a result, the number of elements was twenty. The number of learning data for SVM was ten and that of test data was ten.

Verification performance was evaluated using Equal Error Rate (EER). Error curves are shown in Fig. 5 (a). 


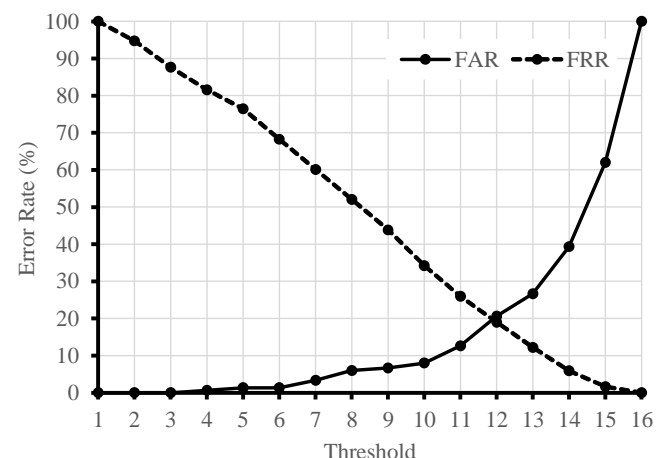

(a)

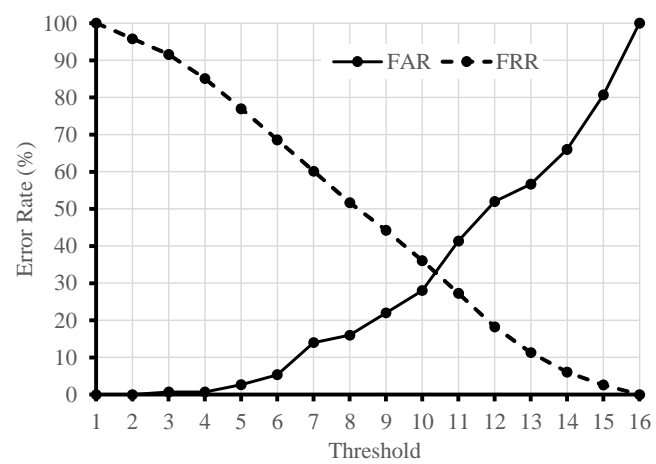

(b)

Figure 5: Error curves, (a): definite signal case and (b): white signal case.

For reference, those when using a white source signal are shown in (b). EER in (a) is $20 \%$ and that in (b) is $30 \%$. It is confirmed that to use a definite source signal instead of a white signal greatly improved verification performance of intra-palm propagation signals. In particular, the FRR curves were almost the same but the FAR curve in the definite signal case was greatly improved comparing with that in the white signal case. This is due to the fact that intraindividual variations were reduced by using the definite signal as a propagation signal.

\section{EFFECT OF PROPAGATION PATH}

In the conventional studies, verification performance of propagated signals on forearms or palms was evaluated. It is expected that other body places may be suitable for person verification using intra-body propagation signals. In this section, verification performances of signals that propagated on another path as shown in Fig. 6 were investigated. The propagation path (a) is on body surface between one's right palm and right wrist, (b) is between one's right and left palms, (c) is between one's right palm and ear, and (d) is between one's right palm and ankle.

In practical applications, body surface regions that are not covered by clothes are suitable for measuring the propagation signals. From the viewpoint, palms are most suitable. The above regions

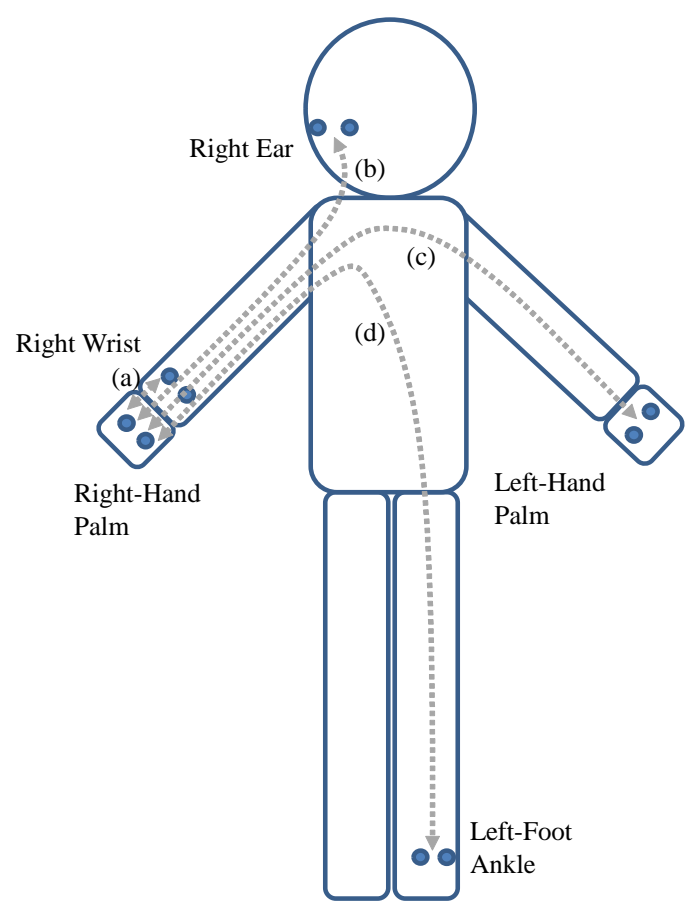

Figure 6: Propagation paths.

Table 2: EERs in various propagation paths.

\begin{tabular}{c|c|c|c|c}
\hline & (i) & (ii) (\%) & (iii) & EER (\%) \\
\hline Right-Hand Palm & 1 & 41.2 & 20 & 20 \\
\hline Right Wrist & 13 & 95.1 & 16 & 24 \\
\hline Right Ear & 17 & 98.0 & 27 & 22 \\
\hline Left-Hand Palm & 11 & 92.6 & 21 & 27 \\
\hline Left-Foot Ankle & 8 & 92.8 & 3 & 25 \\
\hline
\end{tabular}

are not comparatively covered by clothes; therefore, they are also suitable but less suitable than a palm.

For measuring propagated signals on other paths, new devices were prepared as shown in Fig. 7, where, (a), (b), (c), and (d) were for a right wrist, left-hand palm, right ear, and left-foot ankle, respectively. These were used at a receiver side and a conventional device was used for a right-hand palm at a transmitter side.

Measurement conditions were similar to those in Sect. 4.2. However, in the case of measuring left-foot ankle, experimental subjects were standing during measurement.

EERs in four routes are summarized in Table 2, where '(i)', '(ii)', and '(iii)' denote the number of principal components, the value of accumulated proportion in percentage terms, and the number of frequencies, respectively. For reference, results in the case of palm that was presented in the previous sections are also presented.

From these results, it is confirmed that EERs are various in different propagation paths. EER in the case of palm was best but the 

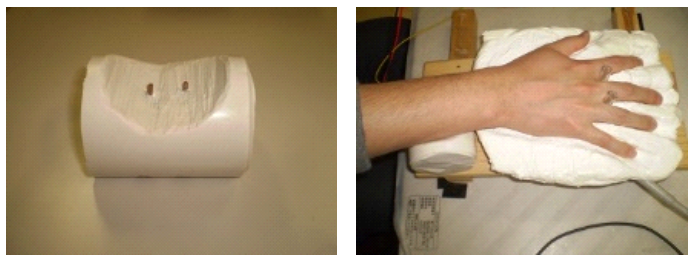

(a)
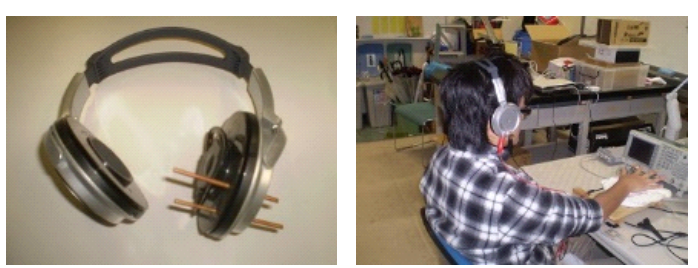

(b)
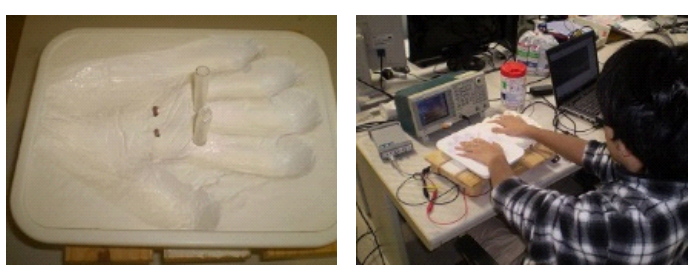

(c)
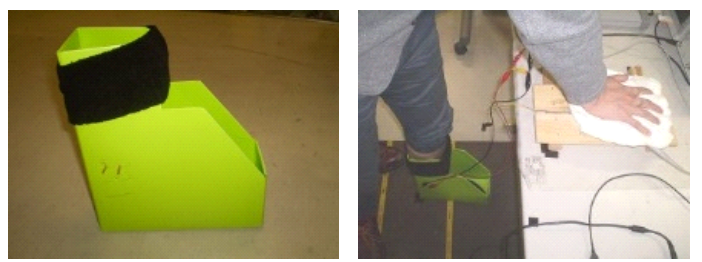

(d)

Figure 7: Devices for measuring other propagation paths.

reason is not clear. It is not true that longer propagation path becomes, better verification performance becomes.

\section{CONCLUSIONS}

In this paper, a definite source signal has been introduced into person verification using intra-body propagation signals and verification performances were evaluated in various propagation paths on the body surface. The effectiveness of using a definite source signal has been confirmed. On the other hand, the knowledge was not obtained that which regions of the body are more suitable for person verification using intra-body propagation signals. A relationship between a region of body and verification performance was not found. The EER in the case of palm that had been used in our conventional study was best.

To use phase spectra as individual features is now being tried in addition to amplitude spectra in order to improve verification performance.

\section{ACKNOWLEDGMENTS}

The authors would like to thank all of the subjects for their collaboration with the measurements.

\section{REFERENCES}

[1] A. Altinok and M. Turk. 2003. Temporal Integration for Continuous Multimodal Biometrics. In Proc. of 2003 Workshop on Multimodal User Authentication (MMUA2003). 207-214.

[2] C. Hsu and C. Lin. 2002. A Comparison of Methods for Multiclass Support Vector Machines. IEEE Trans. Neural Networks 13, 2 (Mar. 2002), 415-425.

[3] T. Inada, Y. Sodani, and I. Nakanishi. 2016. Intra-Palm Propagation Signals as Suitable Biometrics for Successive Authentication. Journal of Computer Technology and Application 7, 2 (Feb. 2016), 65-72.

[4] A. Jain, R. Bolle, and S. Pankanti. 1999. BIOMETRICS Personal Identification in Networked Society. Kluwer Academic Publishers, Massachusetts.

[5] I. Nakanishi, S. Baba, and C. Miyamoto. 2010. On-Demand Biometric Authentication of Computer Users Using Brain Waves. Zavoral F. et al. (Eds.), NDT2010, CCIS 87, I (Jul. 2010), 504-514.

[6] I Nakanishi, T. Inada, and S. Li. 2013. Performance Evaluation of Intra-Palm Propagation Signals as Biometrics. In Proc. of 2013 International Conference on Biometrics and Kansei Engineering (ICBAKE2013). 91-94.

[7] I. Nakanishi, T. Inada, and S. Li. 2014. New Dedicated Measuring Devices for Intra-Palm Propagation Signals. In Proc. of the International Symposium on Biometric and Security Technologies (ISBAST'14). 35-38.

[8] I. Nakanishi and Y. Sodani. 2010. SVM-Based Biometric Authentication Using Intra-Body Propagation Signals. In Proc. of 20107 th IEEE International Conference on Advanced Video and Signal Based Surveillance (AVSS2010).

[9] I. Nakanishi, Y. Sodani, and S. Li. 2013. User Verification Based on the Support Vector Machine Using Intra-Body Propagation Signals. International Journal of Biometrics 5, 3/452 (2013), 288-305.

[10] I. Nakanishi, Y. Yorikane, Y. Itoh, and Y. Fukui. 2007. Biometric Identity Verification Using Intra-Body Propagation Signal. In Proc. of 2007 Biometrics Symposium. 